\title{
Position and frequency shifts induced by massive modes of the gravitational wave background in alternative gravity
}

\author{
Stefano Bellucci ${ }^{1}$ * Salvatore Capozziello ${ }^{2}$ Mariafelicia De Laurentis ${ }^{2}$ 团 and Valerio Faraoni鸠 \\ 1 INFN Laboratori Nazionali di Frascati \\ Via Enrico Fermi 40, I-00044 Frascati, Italy \\ 2 Dip. di Scienze Fisiche, Università di Napoli "Federico II" and INFN Sez. di Napoli \\ Compl. Universitario Monte S. Angelo, Ed. N, Via Cinthia, I-80126 Napoli, Italy and \\ 3 Physics Department, Bishop's University \\ Sherbrooke, Québec, Canada J1M 1Z7
}

\begin{abstract}
Alternative theories of gravity predict the presence of massive scalar, vector, and tensor gravitational wave modes in addition to the standard massless spin 2 graviton of General Relativity (GR). The deflection and frequency shift effects on light from distant sources propagating through a stochastic background of gravitational waves, containing such modes, differ from their counterparts in GR. Such effects are considered as a possible signature for alternative gravity in attempts to detect deviations from Einstein's gravity by astrophysical means.
\end{abstract}

PACS numbers: 04.30, 04.30.Nk, 04.50.+h, 98.70.Vc

\section{INTRODUCTION}

Einstein's theory of GR has been tested in its weakfield approximation and found to pass all the available experiments at terrestrial and Solar System scales [1]. Outside the Solar System, the binary pulsar 1913+16 [2] provides indirect evidence for gravitational waves with an energy loss consistent with GR. However, other theories may produce the same change of orbital parameters: for example, the emission of scalar radiation from this binary within the context of scalar-tensor gravity is necessarily small because, due to the high symmetry of this system, the dipole moment is small. As a consequence, the constraints on scalar-tensor gravity imposed by the binary pulsar are not competitive with those from Solar System tests (however, the binary pulsar data are sufficient to rule out Rosen's bimetric theory) [1].

Gravitational lensing has provided evidence for light deflection on galactic and cluster scales but, due to our ignorance of the detailed mass distribution of the lens, gravitational lens systems constitute poor tests for the theory of gravity (even assuming the validity of GR, the lens model is not unique). Instead, one tries to obtain information about the mass distribution in the lens by assuming the validity of GR and, in this context, obtains evidence for dark matter.

No deviations from Einstein's gravity have been detected so far in the Solar System or binary pulsar and therefore, from the experimental point of view, there is no compelling reason to study alternative gravity theories. On the other hand, high energy theories that incorporate gravity, such as superstring theory, supergravity, and braneworld models, predict deviations from GR in the form of extra scalar, vector, or tensor fields of gravitational origin, massive gravitons, large extra dimensions, higher order corrections to the Einstein equations, or violations of the Equivalence Principle. The low-energy limit of these theories resembles more scalar-tensor or $f(R)$ gravity than GR [3, 4]. This fact, in itself, constitutes a motivation to explore astrophysical and other effects in gravitational theories beyond GR. Further, the 1998 discovery [5] that, if GR is correct, $75 \%$ of the energy content of the universe is in a mysterious and exotic form called dark energy, which propels the accelerated expansion of the universe in the present era [6], leads one to be more inclined towards exploring alternative theories of gravity rather than reinforcing one's faith in Einstein's theory which, after all, has been tested only at the post-Newtonian level and mostly at Solar System scales. It is true that the backreaction of local inhomogeneities in an otherwise Friedmann-Lemaitre-Robertson-Walker universe certainly affects its dynamics, and that this effect is obtained in pure GR without advocating dark energy or modified gravity [7]. However interesting this possibility may be, it has not been possible to produce evidence that the magnitude of this backreaction effect is such that it can explain the cosmic acceleration observed. Backreaction, dark energy, and modified gravity are still open possibilities, each scenario has its own difficulties and, at present no choice between them is compelling, and can be motivated other than by aesthetical considerations or taste. Of course, a conservative relativist could argue that since no deviation from GR has ever been detected, it is pointless to actively pursue competing theories of gravity. On the other hand, proponents of the high energy physics point of view would be justified in replying that we may actually be detecting the first (large scale) deviations from Einstein's theory in the cosmic acceleration, and that it would be foolish to ignore them.

We remind the reader that the need to postulate dark matter in order to explain the rotation curves at galactic and cluster scales, has led people to doubt not only general relativity, but even Newtonian gravity, and has produced MOND and TeVeS theories [8, 9], and $f(R)$ 
gravity with anomalous couplings to matter [10]. Therefore, it seems reasonable to try to identify possible ways to test gravity beyond the Solar System. There seem to be two conflicting points of view, corresponding to two different communities: deviations from Einstein's gravity are regarded as unavoidable by high energy physicists, their detection being only a matter of technological limits. On the other hand, classical relativists may regard the theories producing such deviations as exotica, and it is certainly true that the latter do not have experimental support so far. While theories of gravity alternative to general relativity are purely hypothetical so far, they are theoretically well-motivated and there is scope to try to detect deviations from Einstein's gravity. It is interesting to point out that preliminary results in positive energy theorems exist for such theories as discussed in details in [11]. Besides, in the light of developments in high energy physics, such possibilities should not be discarded a priori. Eventually, experiment is the judge and the failure to detect deviations from GR further constrains alternative theories and worsens the fine-tuning problems that they may have.

Here we do not want to argue in favour of GR or its competitors: rather, we try to bridge the two points of view and we study possible deviations from GR predicted by high energy theories. We focus on a possible astrophysical effect that was studied in the past in the context of GR, and found to be negligible, but is potentially interesting in alternative theories of gravity. This effect consists of the deflection and frequency shift of a light beam due to its propagation through a stochastic background of gravitational waves. In many theories of gravity, extra gravitational fields (scalar, vector, and tensor) appear in addition to the usual massless spin 2 graviton familiar from GR. These modes, massless or massive, correspond to extra degrees of freedom contained in the metric tensor $g_{\mu \nu}$ and show up as gravitational waves emitted by early astrophysical sources or excited by cosmological processes, superposing to form a stochastic background. Such a background, analogous to the cosmic microwave background of electromagnetic waves, is well known in GR, and the propagation of light rays through it has been studied in detail [12, 13, 14, 15, 16, 17]. Consider a pencil of light rays propagating from a distant light source (possibly at a cosmological distance) to an observer. Since gravitational waves deflect light rays and perturb their frequency, naively one expects a photon undergoing $N$ scatterings in this background to be described by a random walk and its deflections, or frequency shifts, to add stochastically as $\sqrt{N}$. Even though the deflection is at most of linear order in the gravitational wave amplitudes which are very small, since the travelled distance can be large, such a cumulative (or " $L$-") effect that grows as $\sqrt{L}$ could compensate for it, and it has indeed been claimed in the past [13, 18]. Intuition fails, however, because it is based on familiarity with random walk processes in which the scatterers are static or nearly static, while the massless gravitons of GR, responsible for photon scattering, propagate at the speed of light. The size of the deflection (or frequency shift) effect is a matter of relative velocities, i.e., of the difference between the speed of the propagating signal and that of the perturbations from a uniform background through which the signal propagates. When this fact is taken into account, the cumulative $L$-effect disappears [14, 15, 16, 17, 19]. The quantitative description of the deflection (or frequency shift) effect depends not only on the relative speed, but also on the spin $s$ of the field responsible for the non-stationary perturbations in the otherwise homogeneous medium. A comprehensive quantitative treatment is given in [19]. The analogous situation for massless scalar modes in scalartensor gravity was briefly considered in [20] and it was found that, in spite of a logarithmic dependence of the rms deflection on $L$, the effect is numerically comparable to the one in GR and, therefore, completely negligible for practical purposes. However, the spectrum of gravitational theories now available is considerably larger and the consideration of astrophysical effects due to massive fields of various spins forming a stochastic background can potentially be of interest, since massive fields can allow for a cumulative $L$-effect, which will be explored in the following sections.

The plan of this paper is as follows. In Sec. II we briefly recall the physics of the deflection and frequency shift effects for non-stationary perturbations of different spins. In Sec. III this analysis is applied to gravitational theories that predict deviations from GR. In Sec. IV the case of modified (or $f(R)$ ) gravity is studied in detail, while Sec. V contains a discussion and the conclusions.

\section{DEFLECTIONS AND FREQUENCY SHIFTS CAUSED BY PROPAGATION IN A GRAVITATIONAL WAVE BACKGROUND}

To realize how gravitational waves induce deflections and frequency shifts in a light ray with tangent $p^{\mu}$ that traverses them, it is sufficient to consider the null geodesic equation

$$
\frac{d p^{\mu}}{d \lambda}+\Gamma_{\alpha \beta}^{\mu} p^{\alpha} p^{\beta}=0 .
$$

By locally expanding the metric as $g_{\mu \nu}=\eta_{\mu \nu}+h_{\mu \nu}$ in an asymptotically Cartesian coordinate system, where the perturbations $h_{\mu \nu}$ (with $\left|h_{\mu \nu}\right|<<1$ ) describe gravitational waves, computing the Christoffel symbols $\Gamma_{\alpha \beta}^{\mu}$ to first order, and using the fact that $p^{\mu}=p_{(0)}^{\mu}+\delta p^{\mu}=$ $(1,0,0,1)+\delta p^{\mu}$ with $\delta p^{\mu}=\mathrm{O}(h)$ for a photon with un- 
perturbed path along the $z$-axis, one obtains

$$
\begin{aligned}
\delta p^{\mu} & =-\int_{S}^{O} d \lambda \Gamma_{\alpha \beta}^{\mu} p_{(0)}^{\alpha} p_{(0)}^{\beta} \\
& =\frac{1}{2} \int_{S}^{O} d z\left(h_{00}-2 h_{03}+h_{33}\right)^{, \mu}+\mathrm{O}\left(h^{2}\right)
\end{aligned}
$$

where the integral is computed along the unperturbed path from the source $S$ to the observer $O$. This shows that, in GR, a gravitational wave propagating (anti)parallel to the light ray has no effect on it, to first order. $^{1}$ If the $h_{\mu \nu}$ describe a superposition of many waves with random phases, directions of propagation, and polarizations, one will obtain deflections such that $\left\langle\delta p^{\mu}\right\rangle=0$ but $\left\langle\left(\delta p^{\mu}\right)^{2}\right\rangle \neq 0$. Therefore the problem is whether these random deflections (for $\mu=1,2,3$ ) or frequency shifts (for $\mu=0$ ) add stochastically. This problem has been solved by Linder [19] in a more general context by considering random fluctuations due to inhomogeneities propagating with arbitrary speed $v$ between the light source (at $z=0$ ) and an observer (at $z=L$ ) and due to a superposition of fields of $\operatorname{spin} s=0,1$, or 2 . By writing the deflection due to a single mode as

$$
\theta_{\mu}=\int_{0}^{L} d z \epsilon_{, \mu}
$$

and $\epsilon(t, \vec{x})=\mathcal{R} e\left(\epsilon_{0} \mathrm{e}^{i k_{\mu} x^{\mu}}\right)$, Linder obtains the mean square deflection

$$
\left\langle\theta_{\mu}^{2}\right\rangle=\frac{1}{2} \Sigma_{s=0}^{2}\left\langle\mathcal{R} e^{2} \epsilon_{s}\right\rangle \sum_{n=-2}^{2 s} a_{n} J_{n},
$$

where $s$ is the spin of the field responsible for the inhomogeneities, $a_{n}$ are constants, and $J_{n}$ are the integrals

$$
J_{n}=\frac{1}{(k L)^{n+1}} \int_{\frac{-k L(1+v)}{2}}^{\frac{k L(1-v)}{2}} d y y^{n} \sin ^{2} y .
$$

One is interested in the limit for wavenumbers $k$ and lenghts $L$ such that $k L>>1$; in this limit the integrals $J_{n}$ for $n \geq 0$ cannot cause an $L$-effect and we focus on the integrals for $n=-2,-1$, given by [19]

$$
\begin{aligned}
& J_{-1}=-4(1+s) v\left(1-v^{2}\right)^{s} \int_{\frac{-k L(1+v)}{2}}^{\frac{k L(1-v)}{2}} d y \frac{\sin ^{2} y}{y} \\
& J_{-2}=k L\left(1-v^{2}\right)^{1+s} \int_{\frac{-k L(1+v)}{2}}^{\frac{k L(1-v)}{2}} d y \frac{\sin ^{2} y}{y^{2}}
\end{aligned}
$$

\footnotetext{
1 This can be seen by adopting the transverse-traceless gauge in which $h_{00}=h_{03}=h_{33}=0$ for a gravitational wave propagating in the $\pm z$ direction.
}

While Linder, in the context of GR, focussed on massless spin 2 gravitons and the limit $v \rightarrow 1$, here we are interested in the opposite limit for massive modes. As shown in the next section, some of these modes can become very massive, corresponding to $v \rightarrow 0$. In this case $J_{-1}$ becomes negligible and we are left with the $J_{-2}$ contribution.

\section{APPLICATION TO ALTERNATIVE THEORIES OF GRAVITY}

In several alternative theories of gravity, massive gravitational fields appear which can potentially give rise to an $L$-effect. Some of them are inspected in the following.

\section{III.1 Scenarios with large extra dimensions}

It has been suggested [21] that the hierarchy problem could be solved in theories with large (sub-millimeter size) extra spatial dimensions, in which gravitons propagate through $(3+n)$-dimensional space while nongravitational physics is confined to the ordinary three spatial dimensions (see [22] for a review). The $n$ extra dimensions are compactified, e.g., on a torus with a radius $R_{n}$ and gravity can be strong already at the TeV scale. The gravitons propagating in the extra dimensions acquire a mass given by

$$
m_{n}^{2}=\frac{4 \pi n^{2}}{R_{n}^{2}},
$$

where

$$
R_{n}=2 \cdot 10^{\frac{32-17 n}{n}} \mathrm{~cm}
$$

The model is ruled out for $n=1$ and marginally ruled out for $n=2$ (for which $R_{2} \sim 2 \mathrm{~mm}$ ), but is viable for $n>2$, corresponding to $R_{n}<10^{-6} \mathrm{~cm}$. The dispersion relation $k_{\mu} k^{\mu}=-m_{n}^{2}$ for the massive gravitons yields the group velocity $v_{g}=\frac{c k}{\sqrt{m_{n}^{2}+k^{2}}}$. If $m_{n}$ is sufficiently large, many (most) modes composing the gravitational wave background will have $k<<m_{n}$ and $v_{g} \sim c k / m_{n}<<c$. For example, for $n=3$, one obtains $m_{3} \sim 3 \cdot 10^{-19 / 3} \mathrm{~cm}^{-1}$; for waves of wavelength $\lambda_{g} \sim 10^{3} \mathrm{~km}$ it is $v_{g} \sim 10^{-2} c$, while longer waves with $\lambda_{g} \sim 3 \cdot 10^{8} \mathrm{~km}=2$ A.U. yield $v_{g} \sim 10^{-7} c$. For $n=4$ and $\lambda_{g} \sim 10^{3} \mathrm{~km}$, it is $v_{g} \sim 10^{-17} \mathrm{c}$.

$$
\text { III.2 } f\left(R, R_{\mu \nu} R^{\mu \nu}, R_{\mu \nu \rho \sigma} R^{\mu \nu \rho \sigma}, \square R, \square^{2} R, \ldots\right) \text { theories }
$$

In general, in theories described by a Lagrangian density of the type $f\left(R, R_{\mu \nu} R^{\mu \nu}, R_{\mu \nu \rho \sigma} R^{\mu \nu \rho \sigma}, \square R, \square^{2} R, \ldots\right), \quad$ there are scalar, vector, and tensor modes, massive or massless, 
and these can, in principle, contribute to the gravitational wave background and produce an $L$-effect. However, some of these massive modes are ghosts, which precludes further consideration of these theories. An exception are theories with Lagrangian of the form $f(R, \mathcal{G})$, where $\mathcal{G}=R^{2}-4 R_{\mu \nu} R^{\mu \nu}+R_{\mu \nu \rho \sigma} R^{\mu \nu \rho \sigma}$ is the Gauss-Bonnet combination. At least if certain conditions are satisfied, ghosts are avoided in these theories [23].

\section{III.3 $N=2,8$ extended supergravity}

The supergravity multiplet in $N=2,8$ extended supergravity contains a graviton, a gravivector field, two Majorana gravitinos for $N=2$, and a graviscalar field for $N=8$. The graviscalar violates the Weak Equivalence Principle [24], and both graviscalar and gravivector are short-ranged. The available experiments set the limits on their ranges $R_{l}$ and $R_{\sigma}$, respectively, [24, 25]

$$
\begin{aligned}
& R_{l} \leq 0.6 \mathrm{~cm}, \quad R_{l} \geq 13 \mathrm{~cm} \quad(N=2), \\
& R_{l} \leq 0.4 \mathrm{~cm}, \quad R_{l} \geq 40 \mathrm{~m} \quad(N=8) \\
& R_{\sigma} \leq 0.15 \mathrm{~cm}, \quad 60 \mathrm{~m} \leq R_{\sigma} \leq 100 \mathrm{~m} .
\end{aligned}
$$

If these fields are truly short-ranged, they can also contribute as massive modes to the gravitational wave background and the analysis of the previous section applies.

There is scope, therefore, to consider the limit $v \rightarrow 0$ for massive gravitons in these scenarios in the discussion of the previous section.

\section{III.4 rms deflections and frequency shifts due to massive modes}

Since $J_{-1} \rightarrow 0$ in the limit of heavy modes $v \rightarrow 0$, we are left with the contribution of

$$
J_{-2} \rightarrow k L \int_{-k L / 2}^{+k L / 2} d y \frac{\sin ^{2} y}{y^{2}}
$$

in eq. (41). By using

$$
\int d y \frac{\sin ^{2} y}{y^{2}}=\frac{\cos (2 y)}{2 y}+\frac{2 y S i(2 y)-1}{2 y},
$$

where $\operatorname{Si}(z) \equiv \int_{0}^{z} d t \frac{\sin t}{t}=\frac{\pi}{2}-\int_{z}^{+\infty} d t \frac{\sin t}{t}$ is the sine integral, one obtains

$$
J_{-2}=2[\cos (k L)+k L S i(k L)-1]
$$

in the $v \rightarrow 0$ limit. The term $2[\cos (k L)-1]$ assumes values in the interval $[-4,0]$ and oscillates as $k L$ becomes large, while the second term $k L S i(k L)$ dominates. Since
$S i(+\infty)=\pi / 2$, the limit $k L>>1$ yields the rms deflection

$$
\sqrt{\left\langle\left(\theta_{\mu}\right)^{2}\right\rangle} \approx \sqrt{\frac{a_{-2} \pi k L}{2} \sum_{s=0}^{2}\left\langle\mathcal{R} e^{2} \epsilon_{s}\right\rangle}
$$

for these modes, where an $L$-effect is indeed present and can, in principle, compensate for small values of the gravitational wave amplitudes $\epsilon_{2}$ to produce a non-negligible effect. This is not surprising since in the limit $v \rightarrow 0$ the propagation of the photon reduces to a random walk.

More precisely, keeping the dependence of $J_{-2}$ on $v$ yields

$$
J_{-2} \simeq 2\left(1-v^{2}\right) k L[S i(k L)+v S i(k L v)]
$$

and

$$
\begin{aligned}
& \sqrt{\left\langle\theta_{\mu}^{2}\right\rangle}= \\
& \sqrt{\sum_{s=0}^{2} 2 a_{-2} k L\left(1-v^{2}\right)[S i(k L)+v S i(k L v)]\left\langle\mathcal{R} e^{2} \epsilon_{s}\right\rangle} .
\end{aligned}
$$

In all the scenarios listed above one can expect very massive modes for which an $L$-effect exists and the rms deflection or frequency shift is given, in order of magnitude, by

$$
\sqrt{\left\langle\theta_{\mu}^{2}\right\rangle} \simeq \sqrt{k L} \epsilon
$$

where $\epsilon$ is the magnitude of the wave amplitude for the massive mode considered. The estimation of this quantity is difficult because it depends on the processes generating the cosmological background, which are subject to much speculation and large uncertainties even in GR. The calculation of precise spectra of gravitational modes in specific processes is beyond the purpose of this work. We assume that detailed studies can provide, in principle, estimates of $\epsilon$ in various frequency bands following assumptions about specific generating processes. In this paper we study in more detail the case of $f(R)$ gravity.

\section{MASSIVE MODES IN $f(R)$ GRAVITY}

Modified or " $f(R)$ " gravity has been proposed recently in order to explain the current acceleration of the universe without resorting to dark energy [26, 27, 28]. $f(R)$ gravity comes in three versions: the metric [26, 27], Palatini [28], and metric-affine [29] formalisms. In the metric formalism, in which the metric tensor is the only independent variable and the connection is the metric connection, the action is

$$
\mathcal{A}=\frac{1}{2 k} \int d^{4} x \sqrt{-g} f(R)+S^{(\text {matter })},
$$


where $f(R)$ is a non-linear function of its argument replacing the usual Einstein-Hilbert Lagrangian $R-2 \Lambda$ ([26, 27] - see [30, 31, 32] for reviews). Corrections to this Lagrangian that become important as $R \rightarrow 0$ can explain the current acceleration of the universe without resorting to dark energy, while early universe physics in a strong curvature regime is instead affected by corrections described by positive powers of $R$. Indeed, the renormalization of GR introduces quadratic corrections [33], a fact that was exploited in Starobinsky's scenario of inflation without scalar fields [34]. The condition $f^{\prime \prime}(R)>0$ is required in the metric (but not in the Palatini) formalism for the absence of tachyons [35, 36] and for non-linear stability [37].

Metric $f(R)$ gravity is dynamically equivalent to an $\omega=0$ Brans-Dicke theory [31, 38 with a non-trivial potential. In fact, by setting $\phi \equiv f^{\prime}(R)$, an equivalent action is $[31,38]$

$$
S=\frac{1}{2 \kappa} \int d^{4} x \sqrt{-g}[\phi R-V(\phi)]+S^{(\text {matter })},
$$

where

$$
V(\phi)=\phi R(\phi)-f(R(\phi)) .
$$

The scalar degree of freedom $f^{\prime}(R)$ satisfies the equation

$$
3 \square \phi+2 V(\phi)-\phi \frac{d V}{d \phi}=\kappa T,
$$

from which one obtains the effective mass 31]

$$
m_{e f f}=\sqrt{\frac{R f^{\prime \prime}(R)-f^{\prime}(R)}{3 f^{\prime \prime}(R)}}
$$

with $R=R(\phi)$.

In metric $f(R)$ cosmology, the dependence of the effective mass of $\phi$ on the curvature and, therefore, on the environmental density is exploited in the chameleon mechanism in order to make these theories viable. At Solar System densities, the scalar has a very short range, thus evading the constraints imposed by Solar System and terrestrial experiments on the equivalent Brans-Dicke theory, while at cosmological densities this range becomes very long and can affect cosmology. This chameleon mechanism (well-known in quintessence models [39]) makes these theories viable, but at the same time it renders the long wavelength scalar modes forming the stochastic background effectively massless. Therefore, the analysis of massless Brans-Dicke scalar modes of Ref. [20] applies and no $L$-effect is present.

It is more interesting, from this point of view, to consider $f(R)$ theories relevant for early universe physics. For example, in the model $f(R)=R+a R^{2}$, it is $m_{\phi}=1 / \sqrt{6 a}$. One expects the parameter $a$ weighting quantum corrections to the Einstein-Hilbert action to be small and, hence, a large mass for the scalar degree of freedom $\phi$, which propagates with group velocity $v_{g} \simeq c k / m_{\phi}=\sqrt{6 a} c k$.

Assuming the conformal transformation

$$
\widetilde{g}_{\mu \nu}=e^{2 \Phi} g_{\mu \nu} \quad \text { with } \quad e^{2 \Phi}=f^{\prime}(R),
$$

where the prime indicates differentiation with respect to the Ricci scalar $R$ and $\Phi$ is the "conformal scalar field", we obtain the conformally equivalent Hilbert-Einstein action

$$
\mathcal{A}=\frac{1}{2 k} \int d^{4} x \sqrt{-\widetilde{g}}[\widetilde{R}+\mathcal{L}(\Phi, \Phi ; \mu)]
$$

where $\mathcal{L}(\Phi, \Phi ; \mu)$ is the conformal scalar field contribution derived from

$$
\widetilde{R}_{\mu \nu}=R_{\mu \nu}+2\left(\Phi_{; \mu} \Phi_{; \nu}-g_{\mu \nu} \Phi_{; \delta} \Phi^{; \delta}-\Phi_{; \mu \nu}-\frac{1}{2} g_{\mu \nu} \Phi_{; \delta}^{; \delta}\right)
$$

and

$$
\widetilde{R}=e^{-2 \Phi}\left(R-6 \square \Phi-6 \Phi_{; \delta} \Phi^{; \delta}\right) .
$$

In any case, as we will see, the $\mathcal{L}(\Phi, \Phi ; \mu)$-term does not affect the gravitational wave tensor equations so it will not be considered further. ${ }^{2}$

Beginning with the action (26) and deriving the Einstein-like conformal equations, the gravitational wave equations expressed in the conformal metric $\widetilde{g}_{\mu \nu}$ are

$$
\widetilde{\square} \widetilde{h}_{i}^{j}=0 .
$$

Since no scalar perturbation couples to the tensor part of the gravitational waves, we have $\delta \Phi=0$ and then

$$
\widetilde{h}_{i}^{j}=\widetilde{g}^{l j} \delta \widetilde{g}_{i l}=e^{-2 \Phi} g^{l j} e^{2 \Phi} \delta g_{i l}=h_{i}^{j}
$$

which means that $h_{i}^{j}$ is a conformal invariant. As a consequence, the plane-wave amplitude defined by $h_{i}^{j}(t, x)=$ $h(t) e_{i}^{j} \exp \left(i k_{l} x^{l}\right)$, where $e_{i}^{j}$ is the polarization tensor, are the same in both metrics. In any case, the d'Alembert operator transforms as

$$
\widetilde{\square}=e^{-2 \Phi}\left(\square+2 \Phi^{; \lambda} \nabla_{\lambda}\right)
$$

and this means that the background is changing while the tensor wave amplitude is not.

In order to study the cosmological stochastic background, the operator (31) can be specified for a Friedmann-Robertson-Walker (FRW) metric given by

$$
d s^{2}=-d t^{2}+a^{2}(t)\left(d x^{2}+d y^{2}+d z^{2}\right),
$$

\footnotetext{
2 Actually, a scalar component of gravitational radiation is often considered [40, 41], but here we are taking into account only the genuine tensor part of the stochastic background.
} 
and then eq. (29) becomes

$$
\ddot{h}+(3 H+2 \dot{\Phi}) \dot{h}+k^{2} a^{-2} h=0,
$$

where $\square=\frac{\partial^{2}}{\partial t^{2}}+3 H \frac{\partial}{\partial t}$ and $k$ is the wave number.

It is worth stressing that eq. (33) applies to any $f(R)$ theory whose conformal transformation can be defined as $e^{2 \Phi}=f^{\prime}(R)$. The solution, i.e., the gravitational wave amplitude, depends on the specific cosmological background (i.e., $a(t)$ ) and the specific theory of gravity (i.e., $\Phi(t))$ [42]. Considering also the conformal time $d \eta=d t / a$, eq. (33) reads

$$
\frac{d^{2} h}{d \eta^{2}}+\frac{2}{\chi} \frac{d \chi}{d \eta} \frac{d h}{d \eta}+k^{2} h=0
$$

where $\chi \equiv a \mathrm{e}^{\Phi}$. Inflation means that $a(t)=a_{0} \exp (H t)$ and then $\eta=\int d t / a=(a H)^{-1}$ and $\frac{d \chi}{\chi d \eta}=-\eta^{-1}$. The exact solution of (34) is

$$
h(\eta)=\sqrt{2} k^{-2}\left[C_{1} \sin k \eta+C_{2} \cos k \eta\right] .
$$

Inside the $H^{-1}$ radius we have $k \eta \gg 1$. Furthermore, considering the absence of gravitons in the initial vacuum state, we have only negative-frequency modes and then the adiabatic behavior is

$$
h=\sqrt{\frac{2}{\pi}} k^{1 / 2} \frac{1}{a H} C \exp (-i k \eta) .
$$

At the first horizon crossing $(a H=k)$, the averaged amplitude of the perturbation $A_{h}=(k / 2 \pi)^{3 / 2}|h|$ is

$$
A_{h}=\frac{C}{2 \pi^{2}} .
$$

When the scale $a / k$ grows larger than the Hubble radius $H^{-1}$, the growing mode of evolution is frozen, that is, it is constant. This situation corresponds to the limit $k \eta \ll 1$ in eq. (35). Since $\Phi$ acts as the inflaton field, it is $\Phi \sim 0$ at re-entry after the end of inflation. Then the amplitude $A_{h}$ of the wave is preserved until the second horizon crossing after which it can be observed, in principle, as an anisotropy perturbation in the cosmic microwave background. It can be shown that $\triangle T / T \lesssim A_{h}$ as an upper limit to $A_{h}$ since other effects can contribute to the background anisotropy [43]. From these considerations, it is clear that the only relevant quantity is the initial amplitude $C$ in eq. (36), which is conserved until re-entry into the horizon. Such an amplitude directly depends on the fundamental mechanism generating the perturbations. Inflation gives rise to processes capable of producing perturbations as zero-point energy fluctuations. Such a mechanism depends on the theory of gravitation adopted and then $(\triangle T / T)$ could constitute a further constraint to select a suitable $f(R)$-theory.
Considering a single graviton in the form of a monochromatic wave, its zero-point amplitude is derived through the equal time commutation relations

$$
\left[h(t, x), \pi_{h}(t, y)\right]=i \delta^{3}(x-y),
$$

where the amplitude $h$ is the field and $\pi_{h}$ is the conjugate momentum operator. Writing the Lagrangian for $h$

$$
\widetilde{\mathcal{L}}=\frac{1}{2} \sqrt{-\widetilde{g}} \widetilde{g}^{\mu \nu} h_{; \mu} h_{; \nu}
$$

in the conformal FRW metric $\widetilde{g}_{\mu \nu}$ ( $h$ is conformally invariant), we obtain

$$
\pi_{h}=\frac{\partial \widetilde{\mathcal{L}}}{\partial \dot{h}}=e^{2 \Phi} a^{3} \dot{h} .
$$

Then, eq. (38) becomes

$$
[h(t, x), \dot{h}(t, y)]=i \frac{\delta^{3}(x-y)}{a^{3} e^{2 \Phi}}
$$

and the fields $h$ and $\dot{h}$ can be expanded in terms of creation and annihilation operators

$$
\begin{aligned}
& h(t, x)=\frac{1}{(2 \pi)^{3 / 2}} \int d^{3} k\left[h(t) e^{-i k x}+h^{*}(t) e^{+i k x}\right], \\
& \dot{h}(t, x)=\frac{1}{(2 \pi)^{3 / 2}} \int d^{3} k\left[\dot{h}(t) e^{-i k x}+\dot{h}^{*}(t) e^{+i k x}\right] .
\end{aligned}
$$

The commutation relations in conformal time are then

$$
\left[h h^{\prime *}-h^{*} h^{\prime}\right]=\frac{i(2 \pi)^{3}}{a^{3} \mathrm{e}^{2 \Phi}} .
$$

The substitution of eqs. (36) and (37) yields $C=$ $\sqrt{2} \pi^{2} H \mathrm{e}^{-\Phi}$, where $H$ and $\Phi$ are calculated at the first horizon-crossing and then

$$
A_{h}=\frac{1}{\sqrt{2}} H \mathrm{e}^{-\Phi},
$$

which means that the amplitude of gravitational waves produced during inflation directly depends on the given $f(R)$ theory since $\Phi=\frac{1}{2} \ln f^{\prime}(R)$. Explicitly, it is [42]

$$
A_{h}=\frac{H}{\sqrt{2 f^{\prime}(R)}},
$$

where $f^{\prime}(R)>0$ is necessary in order for the graviton to carry positive kinetic energy [31]. The representation of $f(R)$ gravity as a Brans-Dicke theory is particularly useful when dealing with the scalar component of gravitational waves, ruled by the equation [44]

$$
\square \Phi=m^{2} \Phi,
$$

where $\Phi \equiv-\delta \phi / \phi_{0}$. The scalar field generates a third component for the tensor polarization of gravitational 
waves and the total perturbation describing a gravitational wave propagating in the positive $z$ direction is

$$
\begin{aligned}
& h_{\mu \nu}(t-z)=A^{+}(t-z) e_{\mu \nu}^{(+)}+A^{\times}(t-z) e_{\mu \nu}^{(\times)} \\
& +\Phi(t-z) e_{\mu \nu}^{(s)} .
\end{aligned}
$$

The term $A^{+}(t-z) e_{\mu \nu}^{(+)}+A^{\times}(t-z) e_{\mu \nu}^{(\times)}$describes the two standard (i.e., tensorial) polarizations of a gravitational wave arising from GR in the TT gauge [45], while the term $\Phi(t-z) e_{\mu \nu}^{(s)}$ is the extension of the TT gauge mode to the scalar case. Three different degrees of freedom are present (see eq.(32) of [41]), while only two are present in standard GR. Then, for a purely scalar gravitational wave, the metric perturbation is [44]

$$
h_{\mu \nu}=\Phi e_{\mu \nu}^{(s)} .
$$

The stochastic background of scalar gravitational waves can be described in terms of the scalar field $\Phi$ and characterized by a dimensionless spectrum (see the analogous definition for tensor modes in [40, 46, 47, 48])

$$
\Omega_{s g w}(f)=\frac{1}{\rho_{c}} \frac{d \rho_{s g w}}{d \ln f},
$$

where

$$
\rho_{c} \equiv \frac{3 H_{0}^{2}}{8 \pi G}
$$

is the (present) critical energy density of the universe, $H_{0}$ is the Hubble parameter today, and $d \rho_{\text {sgw }}$ is the energy density of the scalar gravitational radiation in the frequency interval $(f, f+d f)$. We are now using standard units. Now it is possible to write an expression for the energy density of the stochastic scalar relic gravitons background in the angular frequency interval $(\omega, \omega+d \omega)$ as

$d \rho_{s g w}=2 \hbar \omega\left(\frac{\omega^{2} d \omega}{2 \pi^{2} c^{3}}\right) N_{\omega}=\frac{\hbar H_{d S}^{2} H_{0}^{2}}{4 \pi^{2} c^{3}} \frac{d \omega}{\omega}=\frac{\hbar H_{d S}^{2} H_{0}^{2}}{4 \pi^{2} c^{3}} \frac{d f}{f}$,

where $f$, as above, is the frequency in standard comoving time. Eq. (52) can be rewritten in terms of the critical and de Sitter energy densities

$$
H_{0}^{2}=\frac{8 \pi G \rho_{c}}{3 c^{2}}, \quad H_{d S}=\frac{8 \pi G \rho_{d S}}{3 c^{2}} .
$$

Introducing the Planck density $\rho_{\text {Planck }}=\frac{c^{5}}{\hbar G^{2}}$, the spectrum is given by

$$
\Omega_{s g w}(f)=\frac{1}{\rho_{c}} \frac{d \rho_{s g w}}{d \ln f}=\frac{f}{\rho_{c}} \frac{d \rho_{s g w}}{d f}=\frac{16}{9} \frac{\rho_{d S}}{\rho_{\text {Planck }}} .
$$

At this point, some comments are in order. First, the calculation works for a simplified model that does not include the matter-dominated era. If the latter is included, the redshift at the equivalence epoch has to be considered. Taking into account Ref. [49], one gets

$$
\Omega_{\text {sgw }}(f)=\frac{16}{9} \frac{\rho_{d S}}{\rho_{\text {Planck }}}\left(1+z_{e q}\right)^{-1}
$$

for the waves which, at the epoch in which the universe becomes matter-dominated, have a frequency higher than $H_{e q}$, the Hubble parameter at equivalence. This situation corresponds to frequencies $f>\left(1+z_{e q}\right)^{1 / 2} H_{0}$ today. The redshift correction in eq. (55) is needed since the present value of the Hubble parameter $H_{0}$ would be different without a matter-dominated contribution. At lower frequencies, the spectrum is given by [46, 48]

$$
\Omega_{\text {sgw }}(f) \propto f^{-2} .
$$

As a further consideration, let us note that the results (54) and (55), which are frequency-independent, do not hold in the entire range of physical frequencies. For waves with frequencies less than the present Hubble parameter $H_{0}$, the notion of energy density is not defined because the wavelength becomes longer than the Hubble scale. Similarly, at high frequencies, there is a maximal frequency above which the spectrum rapidly drops to zero. In the above calculation, the simplifying assumption that the phase transition from the inflationary to the radiation dominated epoch is instantaneous has been made. In the physical universe, this process occurs over some time scale $\Delta \tau$, with

$$
f_{\max }=\frac{a\left(t_{1}\right)}{a\left(t_{0}\right)} \frac{1}{\Delta \tau},
$$

which is the redshifted rate of the transition. In any case, $\Omega_{\text {sgw }}$ drops rapidly. The two cutoffs at low and high frequencies for the spectrum guarantee that the total energy density of the relic scalar gravitons is finite. For GUT-scale inflation, it is of the order [46]

$$
\frac{\rho_{\text {ds }}}{\rho_{\text {Planck }}} \approx 10^{-12} \text {. }
$$

These results can be quantitatively constrained considering the recent $W M A P$ release. In fact, it is well known that WMAP observations put severe restrictions on the spectrum. In Fig. 1 the spectrum $\Omega_{\text {sgw }}$ is mapped: considering the ratio $\rho_{d s} / \rho_{\text {Planck }}$, the relic scalar gravitational wave spectrum seems consistent with the WMAP constraints on scalar perturbations. Nevertheless, since the spectrum falls off as $f^{-2}$ at low frequencies, today at $L I G O / V I R G O$ and LISA frequencies (indicated in Fig. 1), one gets

$$
\Omega_{\text {sgw }}(f) h_{100}^{2}<2.3 \times 10^{-12},
$$

where $h_{100}=H_{0} /\left(100 \mathrm{~km} \cdot \mathrm{s}^{-1} \cdot \mathrm{Mpc}^{-1}\right)$. It is interesting to calculate the corresponding strain at $f \sim 100 \mathrm{~Hz}$, where interferometers such as VIRGO and LIGO achieve 
maximum sensitivity. The well known equation for the characteristic amplitude [46, 48] adapted to the scalar component of gravitational waves

$$
\Phi_{c}(f) \simeq 1.26 \times 10^{-18}\left(\frac{1 \mathrm{~Hz}}{f}\right) \sqrt{h_{100}^{2} \Omega_{s g w}(f)},
$$

can be used to obtain

$$
\Phi_{c}(100 \mathrm{~Hz})<2 \cdot 10^{-26} .
$$

Then, since we expect a sensitivity of the order of $10^{-22}$ for the above interferometers at $f \sim 100 \mathrm{~Hz}$, we need to gain four orders of magnitude. Let us analyze the situation also at lower frequencies. The sensitivity of the VIRGO interferometer is of the order of $10^{-21}$ at $f \sim 10 \mathrm{~Hz}$ and in that case it is

$$
\Phi_{c}(10 \mathrm{~Hz})<2 \cdot 10^{-25} .
$$

The sensitivity of the LISA interferometer will be of the order of $10^{-22}$ at $f \sim 10^{-3} \mathrm{~Hz}$ and in this case it is

$$
\Phi_{c}\left(10^{-3} \mathrm{~Hz}\right)<2 \cdot 10^{-21} .
$$

This means that a stochastic background of relic scalar gravitational waves could, in principle, be detected by the LISA interferometer.

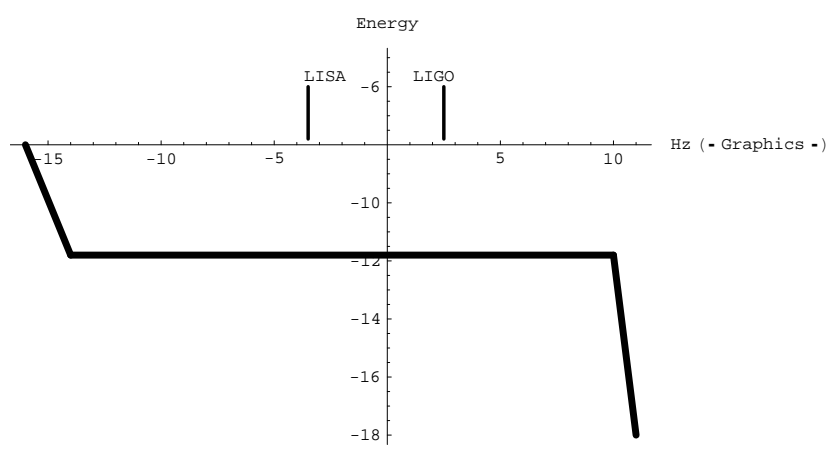

Figure 1: The spectrum of relic scalar gravitational waves in inflationary models is flat over a wide range of frequencies. The horizontal axis is $\log _{10}$ of frequency, in $\mathrm{Hz}$. The vertical axis is $\log _{10} \Omega_{g s w}$. The inflationary spectrum rises quickly at low frequencies (wave which re-entered the Hubble sphere after the universe became matter-dominated) and falls off above the (appropriately redshifted) frequency scale $f_{\max }$ associated with the fastest characteristic time of the phase transition at the end of inflation. The amplitude of the flat region depends only on the energy density during the inflationary stage; we have chosen the largest amplitude consistent with the WMAP constraints on scalar perturbations. This means that, at $L I G O$ and $L I S A$ frequencies, $\Omega_{\text {sgw }}<2.3 \cdot 10^{-12}$.

To estimate the rms deflection effect from massive scalar modes in the gravitational wave background, we restrict to periods that are of the order of hours or days.
A longer period would result in a "frozen" effect which is much less likely to be detected, while a much shorter period would probably render the deflections unobservable because only an averaged position shift would be recorded during observation times longer than the period itself and a slightly blurred image would be the outcome (although fast photometry might allow to push the limits). Assuming a frequency $\sim 10^{-3} \mathrm{~Hz}$ at a distance $L \sim 500 \mathrm{kpc}$ and using the upper limit (63), one obtains a rms deflection $\theta_{r m s} \sim \sqrt{k L} \epsilon \sim 10^{-10}$. The maximum resolution expected with high precision astrometry is of the order of microarcseconds ( $\sim 10^{-7}$ radians), three orders of magnitude above the required sensitivity for detection. For galactic sources at $L \sim 5 \mathrm{kpc}$, to which high precision astrometry is more likely to apply, $\theta_{r m s}$ drops by another order of magnitude. One can, of course, consider different sources of electromagnetic radiation with slightly higher frequency, at more promising distances $L$, and perhaps find mechanism which produce higher scalar amplitudes $\Phi$ : at a first look, however, it is unlikely that the four orders of magnitude necessary for detection can be bridged in the foreseeable future.

\section{OUTLOOKS}

All modern theories of high energy physics unifying gravity with the other interactions predict departures from GR; however, no such deviation has been observed so far in Solar System experiments, and practically all the experimental constraints on such deviations are obtained within the Solar System (the binary pulsar and gravitational lensing provide constraints that are not competitive with those obtained from Solar System experiments). Therefore, it is interesting to explore astrophysical effects outside of this narrow region of the universe that could potentially exhibit deviations from Einstein's theory. The cumulative deflections, or frequency shifts due to propagation of light from distant sources through random massive modes of the gravitational wave background could constitute such an effect. There is now a wide range of theories predicting massive scalar, vector, and tensor modes that can lead to such an effect. However, the astrophysical and cosmological processes generating cosmological gravitational wave backgrounds in these theories are still unexplored. Here we do not attempt to estimate the average strength $\epsilon$ of the various modes appearing in these theories of gravity, in different ranges of wavelengths, and under various assumptions. We limit ourselves to outline an estimate for a particular case: massive scalar modes in $f(R)$ gravity. This class of theories has been the subject of much recent attention in order to explain the observed acceleration of the universe without resorting to dark energy. For the situation considered here, the deflections seem to be a few orders of magnitude too small for detection; on the other 
hand, we find that these scalar modes are certainly of interest for direct attempts at detection with the LISA experiment. According to our preliminary discussion, the indirect detection with the position and frequency shift effect does not seem to be feasible with current technology; however, a more detailed analysis is necessary before definitive conclusions can be drawn. It is, in principle, possible that massive gravitational wave modes could be produced in more significant quantities in cosmological or early astrophysical processes in alternative theories of gravity - the latter are still unexplored. This possibility should be kept in mind when looking for a signature distinguishing these theories from GR, and seems to deserve further investigation.

VF acknowledges the Natural Sciences and Engineering Research Council of Canada for financial support and INFN-Laboratori Nazionali di Frascati, the University of Naples "Federico II", and the International School for Advanced Studies in Trieste for their hospitality.

* bellucci@lnf.infn.it

† capozziello@na.infn.it

‡ felicia@na.infn.it

$\S$ vfaraoni@ubishops.ca

[1] C.M. Will 1993, Theory and Experiment in Gravitational Physics (CUP, Cambridge).

[2] R.A. Hulse and J.H. Taylor 1975, Astrophys. J. Lett. 195, L51.

[3] C.G. Callan, D. Friedan, E.J. Martinez, and M.J. Perry 1985, Nucl. Phys. B 262, 593; E.S. Fradkin and A.A. Tseytlin 1985, Nucl. Phys. B 261, 1.

[4] K.S. Stelle 1978, Gen. Rel. Gravit. 9, 353.

[5] A.G. Riess et al. 1998, Astron. J. 116, 1009; 1999, Astron. J. 118, 2668; 2001, Astrophys. J. 560, 49; 2004, Astrophys. J. 607, 665; S. Perlmutter et al. 1998, Nature 391, 51; 1999, Astrophys. J. 517, 565; 2003, J.L. Tonry et al., Astrophys. J. 594, 1; R. Knop et al. 2003, Astrophys. J. 598, 102; B. Barris et al. 2004, Astrophys. J. 602, 571 .

[6] E.V. Linder, Am. J. Phys. 76, 197 (2008).

[7] T. Buchert 2000, Gen. Rel. Grav. 32, 105; 2001, Gen. Rel. Grav. 33, 1381; 2008, Gen. Rel. Grav. 40, 467; T. Buchert, J. Larena, and J.-M. Alimi 2006, Class. Quantum Grav. 23, 6379; D. Wiltshire 2007, New J. phys. 9, 377; A. Paranjape 2008, Phys. Rev. D 78, 063522; arXiv:0811.2619, E. Kolb, V. Marra, and S. Matarrese 2008, Phys. Rev> D 78, 103002; and references therein.

[8] M. Milgrom 1983, Astrophys. J. 270, 365; J. Bekenstein and M. Milgrom 1984, Astrophys. J. 286, 7; M. Milgrom 2002, New Astr. Rev. 46, 741; 2003, Astrophys. J. 599, $\mathrm{L} 25$.

[9] J.D. Bekenstein 2004, Phys. Rev. D 70, 083509.

[10] S. Nojiri and S.D. Odintsov 2004, Phys. Lett. B 599, 137; G. allemandi, A Borowiec, M. Francaviglia and S.D. Odintsov 2005, Phys. Rev. D 72, 063505; O. Bertolami, C.G. Böhmer, T. Harko, and F.S.N. Lobo 2007, Phys. Rev. D 75, 104016; arXiv:0811.2876; V. Faraoni 2007, Phys. Rev. D 76, 127501; T.P. Sotiriou and V. Faraoni
2008, Class. Quant. Grav. 25, 205002

[11] H.-J. Schmidt 1987, Ann. der Physik 44, 361.

[12] J.A. Wheeler 1960, in Rendiconti della Scuola Internazionale di Fisica "Enrico Fermi", 11th Course of the Varenna Summer School, 1959,"Interazioni Deboli". L.A. Radicati (Ed.). Zanichelli, Bologna. p. 67; W.J. Kaufmann 1970, Nature 227, 157; A.V. Korotun 1970, Sov. Astron. 24, 273; W.L. Burke 1975, Astrophys. J. 196, 329; 1981, Astrophys. J. 244, L1; B. McBreen and L. Metcalfe 1988, Nature 332, 234; B. Allen 1989, Phys. Rev. Lett. 63, 2017; 1990, Gen. Rel. Grav. 22, 1447; V.B. Braginsky, N.S. Kardashev, A.G. Polnarev and I. Novikov 1990, N. Cimento 105B, 1141; I. Kovner 1990, Astrophys. J. 351, 114; P.P. Kronberg, C.C. Dyer, E.M. Burbidge, and V.T. Junkkarinen 1991, Astrophys. J. 367, L1; C.C. Dyer and E.G. Shaver 1992, Astrophys. J. 390, L5; V. Faraoni 1992, Astrophys. J. 398, 425; 1993, in Gravitational Lenses, Proceedings, Hamburg 1991, R. Kayser, T. Schramm and S. Refsdal eds. (Springer, Berlin); 1993, Astron. Astrophys. 272, 385; 1996, Astr. Lett. Comm. 35, 305; 1998, Int. J. Mod. Phys. D 7, 409; 2008, New Astron. 13, 178; A. Labeyrie 1993, Astron. Astrophys. 268, 823; R. Fakir 1993, Astrophys. J. 418, 202; arXiv:gr-qc/9309021; 1994, Phys. Rev. D 50, 3795; 1994, Astrophys. J. 426, 74; 1996, arXiv:astro-ph/9601127, 1997, Int. J. Mod. Phys. D 6, 49; R. Durrer 1994, Phys. Rev. Lett. 72, 3301; D.D. Harari and G.C. Surpi 1994, Phys. Rev. D 50, 4895; S. Pogrebenko et al. 1994, Proc. 2nd EVN/JIVE Symposium, Torun, Poland 1994, A.J. Kus, R.T. Schilizzi, K.M. Borkowski and L.I. Gurvits eds. (Torun Radio Astronomy Observatory, Torun, Poland), p. 33; 1994, Abstracts XXIInd GA IAU Meeting. Twin Press, Netherlands, p. 105; 1996, in Proc. IAU Symp. 165, Compact Stars in Binaries, The Hague, Netherlands, 1994, J. Van Paradijs, E.P.J. Van den Heuvel and E. Kuulkers eds. (Kluwer, Dordrecht), p. 546; R. Bar-Kana 1996, Phys. Rev. D 54, 7138; T. Pyne, C.R. Gwinn, M. Birkinshaw, T.M. Eubanks, and D.N. Matsakis 1996, Astrophys. J. 465, 566; C. Bracco 1997, Astron. Astrophys. 321, 985; C. Bracco and P. Teyssandier 1998, ibidem 339, 921; C.R. Gwinn, T.M. Eubanks, M. Birkinshaw and D.N. Matsakis 1997, Astrophys. J. 485, 87; N. Kaiser and A. Jaffe 1997, Astrophys. J. 484, 545; T. Damour and G. Esposito-Farèse 1998, Phys. Rev. D 58, 044003; J.A. Frieman, G.C. Surpi and D.D. Harari 1999, Astrophys. J. 515, 455; S.M. Kopeikin, G. Schafer, C.R. Gwinn, and T.M. Eubanks 1999, Phys. Rev. D 59, 084023; S.L. Larson and R. Schild, arXiv:astro-ph/0007142, S. Kopeikin and B. Mashhoon 2002, Phys. Rev. D 65, 064025; A.R. Prasanna and S. Mohanty 2002, Europhys. Lett. 60, 651; R. Ragazzoni, G. Valente, and E. Marchetti 2003, Mon. Not. R. Astron. Soc. 345, 100; S.M. Kopeikin and P. Korobkov, arXiv:gr-qc/0510084 G.B. Lesovik, A.V. Lebedev, V. Mounutcharyan, and T. Martin 2005, Phys. Rev. $D$ 71, 122001; S.M. Kopeikin, P. Korobkov, and A.G. Polnarev 2006, Class. Quantum Grav. 23, 4299; R. Fakir and W.G. Unruh, arXiv:0805.3750.

[13] F. Winterberg 1968, Nuovo Cimento 53 B, 1096.

[14] D.M. Zipoy 1966, Phys. Rev. 142, 825.

[15] D.M. Zipoy and B. Bertotti 1968, Nuovo Cimento, 56 B, 195.

[16] B. Bertotti and R. Catenacci 1975, Gen. Rel. Grav. 6, 329. 
[17] G. Dautcourt 1969, Mon. Not, R. Astr. Soc. 144, 255; 1974, in Proc. IAU Symp. 63, Confrontation of Cosmological Theories with Observation, M.S. Longair ed. (Reidel, Dordrecht), p. 299; 1975, Astron. Astrophys. 38, 344; 1975, Astron. Nachr. 298, 81.

[18] F.R. Marleau and G.D. Starkman, arXiv:astro-ph/9605066

[19] E.V. Linder 1986, Phys. Rev. D 34, 1759.

[20] V. Faraoni and E. Gunzig 1998, Astron. Astrophys. 332, 1154.

[21] N. Arkani-Hamed, S. Dimopoulos, and G.R. Dvali 1998, Phys. Lett. B 429, 263.

[22] V.H.S. Kumar and P.K. Suresh, arXiv:hep-th/0606194

[23] G. Calcagni, B. de Carlos, and A.De Felice 2006, Nucl. Phys. B 752, 404; A. De Felice, M. Hindmarsh, and M. Trodden 2006, J. Cosmol. Astropart. Phys. 08:005.

[24] J. Scherk 1979, Phys. Lett. B 88, 265; 1979, in Supergravity, Proceedings of the 1979 Supergravity Workshop at Stony Brook, P. van Nieuwenhuizen and D.Z. Freedman eds. (North-Holland, Amsterdam) p. 43.

[25] S. Bellucci and V. Faraoni 1994, Phys. Rev. D 49, 2922; 1996, Phys. Lett. B 377, 55; Experimental limits on antigravity in extended supergravity. LNF-96-054-P, Oct 1996. Presented at the Congresso Nazionale Societa Italiana di Fisica, Verona, Italy, 23-28 Sep 1996.

[26] S. Capozziello 2002, Int. J. Mod. Phys. D 11, 483; S. Capozziello, S. Carloni, and A. Troisi, arXiv:astro-ph/0303041

[27] S.M. Carroll, V. Duvvuri, M. Trodden, and M.S. Turner 2004, Phys. Rev. D 70, 043528.

[28] D.N. Vollick 2003, Phys. Rev. D 68, 063510.

[29] T.P. Sotiriou 2006, Class. Quantum Grav. 23, 5117; arXiv:gr-qc/0611158, arXiv:0710.4438, T.P. Sotiriou and S. Liberati 2007, Ann. Phys. (NY) 322, 935; 2008, J. Phys. Conf. Ser. 68, 012022.

[30] S. Capozziello and M. Francaviglia 2008, Gen. Rel. Grav. 40, 357.

[31] T.P. Sotiriou and V. Faraoni, arXiv:0805.1726

[32] S. Nojiri and S.D. Odintsov 2007, Int. J. Geom. Meth. Mod. Phys. 4, 115; N. Straumann, arXiv:0809.5148, H.-J. Schmidt 2007, Int. J. Geom. Meth. Phys. 4, 209 (arXiv:gr-qc/0602017); V. Faraoni, arXiv:0810.2602 T.P. Sotiriou, arXiv:0805.1726
[33] R. Utiyama and B. DeWitt 1962, J. Math. Phys 3, 608; K.S. Stelle 1977, Phys. Rev. D 16, 953; A. Strominger 1984, Phys. Rev. D 30, 2257; I.L. Buchbinder, S.D. Odintsov, and I.L. Shapiro 1992, Effective Action in Quantum Gravity (IOP, Bristol); G. Vilkovisky 1992, Class. Quantum Grav. 9, 985.

[34] A.A. Starobinsky 1980, Phys. Lett. B 91, 99.

[35] A.D. Dolgov and M. Kawasaki 2003, Phys. Lett. B 573, 1; S. Nojiri and S.D. Odintsov 2003, Phys. Rev. D 68, 123512.

[36] V. Faraoni 2006, Phys. Rev. D 74, 104017.

[37] F. Briscese, E. Elizalde, S. Nojiri and S. D. Odintsov 2007, Phys. Lett. B 646, 105; A.V. Frolov 2008, Phys. Rev. Lett. 101, 061103; A.A. Appleby and R.A. Battye 2008, Phys. Lett. B 654, 7; S. Nojiri and S.D. Odintsov 2008, Phys. Rev. D 78, 046006.

[38] P.W. Higgs 1959, Nuovo Cimento 11, 816; B. Whitt 1984, Phys. Lett. B 145, 176; P. Teyssandier and P. Tourrenc 1983, J. Math. Phys. 24, 2793; J.D. Barrow and S. Cotsakis 1988, Phys. Lett. B 214, 515; K. Maeda 1989, Phys. Rev. D 39, 3159; T. Chiba 2005, Phys. Lett. B 575, 1.

[39] J. Khoury and A. Weltman 2004, Phys. Rev. Lett., 93, 171104; Phys. Rev. D 69, 044026.

[40] M. Maggiore 2000, Phys. Rep. 331, 283.

[41] S. Capozziello and C. Corda 2006, Int. J. Mod. Phys. D 15, 1119.

[42] S. Capozziello, C. Corda, and M. De Laurentis 2007, Mod. Phys. Lett. A 22, 1097.

[43] A.A. Starobinsky 1982, Sov. Phys. Lett. (JETP) 34, 438.

[44] S. Capozziello, C. Corda, and M. De Laurentis 2007, Mod. Phys. Lett. A 22, 2647.

[45] C.W. Misner, K.S. Thorne and J.A. Wheeler, Gravitation (Freeman, San Francisco, 1973).

[46] B. Allen, in Proceedings of the Les Houches School on Astrophysical Sources of Gravitational Waves, eds. J.A. Marck and J.-P. Lasota (CUP, Cambridge, England 1998).

[47] B. Allen and A.C. Ottewill 1997, Phys. Rev. D 56, 545.

[48] L. Grishchuk et al. 2001, Sov. Phys. Usp. 44, 1; 2001, Usp. Fiz. Nauk 171, 3.

[49] B. Allen 1988, Phys. Rev. D 3, 2078. 